(6) OPEN ACCESS

Additional material is published online only. To view please visit the journal online (http://dx.doi.org/10.1136/ gutjnl-2019-318227).

Center for Interventional Endoscopy, AdventHealth Orlando, Orlando, Florida, USA

Correspondence to Dr Shyam Varadarajulu, Center for Interventional Endoscopy, AdventHealth Orlando, Orlando, FL 32803, USA; svaradarajulu@yahoo.com

Received 6 January 2019 Revised 22 January 2019 Accepted 1 February 2019 Published Online First 12 February 2019
Check for updates

(C) Author(s) (or their employer(s)) 2019. Re-use permitted under CC BY-NC. No commercial re-use. See rights and permissions. Published by BMJ.

To cite: Bang JY, Sutton B, Hawes $\mathrm{R}$, et al. Gut

2019:68:1915-1917.

\title{
Concept of disposable duodenoscope: at what cost?
}

\author{
Ji Young Bang, Bryce Sutton, Robert Hawes, Shyam Varadarajulu ${ }^{\bullet}$
}

\section{MESSAGE}

Although most postendoscopic retrograde cholangiopancreatography (ERCP) biliary tract infections are attributed to suboptimal ductal drainage, transmission of infection by contaminated reusable duodenoscopes has been reported. To overcome this limitation, a disposable duodenoscope has been recently developed. However, given the widespread use and large volume of ERCPs being performed worldwide, the financial viability of this novel concept is unclear. Utilising an activity-based costing and financial model, we estimated that the per-procedure cost of a disposable duodenoscope in the United States can vary from \$797 to \$1547 for centres performing at the $75^{\text {th }}$ percentile of ERCP procedure volume and from $\$ 1318$ to $\$ 2068$ for centres performing at the $25^{\text {th }}$ percentile of procedure volume, based on infection rates of $0.4 \%$ to $1 \%$, respectively. However, when infection was not factored, the per-procedure cost decreased to $\$ 818$ and $\$ 297$ for centres performing at the $25^{\text {th }}$ and $75^{\text {th }}$ percentiles, respectively.

\section{IN MORE DETAIL}

Given the recent reports on infection outbreaks, including carbapenem-resistant Enterobacteriaceae (CRE), caused by contaminated duodenoscopes used at ERCP, ${ }^{1-4}$ a disposable duodenoscope (Exalt $^{\mathrm{TM}}$, Single-Use Duodenoscope, Boston Scientific Corporation, Natick, Massachusetts, USA) has been developed to circumvent this problem. ${ }^{5}$ To determine the adaptability of this innovation in clinical practice, we estimated the per-procedure cost of ERCP using a reusable duodenoscope in order to assess the break-even costs for transitioning to disposable duodenoscopes. An activity-based costing and financial model was constructed based on the current clinical practice of ERCP at a tertiary medical centre in the USA.

The annual procedure volume was 1752 ERCPs in 2018. Nine duodenoscopes with lifetime usage of 3 years and a reprocessing turn-over time of $40 \mathrm{~min}$ per scope were required to meet the clinical needs. The cost of an individual duodenoscope was \$35000 (\$11 667/year) with annual maintenance and repair costs of $\$ 1451$ per scope. The annual cost of the scope washer, inclusive of its maintenance, was $\$ 8000$. The annual cost of scope cleaning supplies and filters was $\$ 10424$, and cleaning-related labour costs including fringe benefits amounted to $\$ 60230$. Based on the published literature, the range of duodenoscope-related infection was estimated at $0.4 \%-1 \%$, and the treatment cost per case of cholangitis at our institution was $\$ 125000$ that included a two-night stay in the intensive care unit. $^{1-3}$ For modelling purposes, the two types of duodenoscopes were assumed to have equal functionality, and only duodenoscope-related (cleaning, repairs, maintenance and infection) costs were factored.

Using data from activity-based costing, financial models and published literature, the estimated per-procedure cost of reusable duodenoscopes varied from $\$ 612$ to $\$ 1362$ based on infection rates of $0.4 \%-1 \%$, respectively. However, when infection was not factored in the analysis, the per-procedure cost dropped to $\$ 112$. Table 1 and figure 1 show the estimated costs based on procedure volume and infection rates and online supplementary tables 1 and 2 are worksheets of estimated costs based on the presence or absence of infection. For institutions performing at the 25 th percentile of USA ERCP procedural volume ( $\leq 50 \mathrm{ERCPs}$ ) year), the cost per procedure varied from $\$ 1318$ to $\$ 2068$ when infection rates increased from $0.4 \%$ to $1 \%$, respectively; however, when infection was not factored, the cost decreased to $\$ 818$. For institutions performing at the 75 th percentile (125-150 ERCPs/ year), the estimated cost per procedure ranged from $\$ 797$ to $\$ 1547$ when infection rates increased from $0.4 \%$ to $1 \%$, respectively; when infection was not factored, the cost decreased to $\$ 297$.

\section{COMMENTS}

Our analysis suggests that the cost of a disposable duodenoscope varies depending on infection rates and ERCP procedure volume. For low-volume centres ( $\leq 50 \mathrm{ERCPs} / \mathrm{year}$ ), the break-even cost for a disposable duodenoscope was $\geq \$ 1300$ depending on infection rates. For large-volume centres ( $\geq 150$ ERCPs/year), the break-even cost was $\geq \$ 800$. The difference in costs is attributed to the fixed costs of investment in capital equipment, maintenance, supplies, personnel and treatment cost of infection which could be offset by the use of disposable scopes at low volume centres. However, at large volume centres, these investment costs are distributed over a higher volume, lowering the mean cost per procedure. For a large-volume centre with a lower break-even cost, disposable duodenoscopes would have to be priced much lower to break even on cost.

Outbreaks of duodenoscope-associated infection have been reported with organisms that include Klebsiella pneumoniae, Pseudomonas aeruginosa and more recently CRE. ${ }^{2-4}$ Unlike standard endoscopes, the duodenoscope is a complex instrument whose structural design includes a recessed space containing an elevator, elevator cable and channel. Because the elevator is recessed and has a complex surface pattern, it is difficult to clean and has been incriminated as a major source of infection in recent 
Table 1 Mean cost of duodenoscopes per procedure (US \$) according to infection rate and annual ERCP volume

\begin{tabular}{|c|c|c|c|c|c|c|c|c|c|c|c|c|c|}
\hline & \multicolumn{13}{|c|}{ Total no. of ERCPs performed per year } \\
\hline & 25 & 50 & 100 & 150 & 200 & 250 & 500 & 750 & 1000 & 1250 & 1500 & 1750 & 2000 \\
\hline \multicolumn{14}{|c|}{ Infection rate (\%) } \\
\hline 0 & 1599 & 818 & 427 & 297 & 232 & 240 & 162 & 135 & 122 & 124 & 117 & 112 & 109 \\
\hline 0.4 & 2099 & 1318 & 927 & 797 & 732 & 740 & 662 & 635 & 622 & 624 & 617 & 612 & 609 \\
\hline 0.6 & 2349 & 1568 & 1177 & 1047 & 982 & 990 & 912 & 885 & 872 & 874 & 867 & 862 & 859 \\
\hline 0.8 & 2599 & 1818 & 1427 & 1297 & 1232 & 1240 & 1162 & 1135 & 1122 & 1124 & 1117 & 1112 & 1109 \\
\hline 1 & 2849 & 2068 & 1677 & 1547 & 1482 & 1490 & 1412 & 1385 & 1372 & 1374 & 1367 & 1362 & 1359 \\
\hline 1.5 & 3474 & 2693 & 2302 & 2172 & 2107 & 2115 & 2037 & 2010 & 1997 & 1999 & 1992 & 1987 & 1984 \\
\hline
\end{tabular}

ERCP: endoscopic retrograde cholangiopancreatography.

outbreaks. Persistent bacterial growth noted on some duodenoscopes is attributed to the presence of a biofilm, which protects the organisms from gas or liquid disinfection. ${ }^{6}$ Biofilm, once formed, must be mechanically removed or dissolved to prevent infection transmission. This issue is not unique to a specific manufacturer or model: culture studies demonstrate that duodenoscopes from all three major manufacturers, including multiple models, have tested positive for infection and biofilm formation. In a nationwide prevalence study from the Netherlands, one-fifth of sampled duodenoscopes were contaminated with any microorganism ( $\geq 20$ colony-forming units $/ 20 \mathrm{~mL}$ ), and the presence of microorganisms with gastrointestinal or oral origin was detected in $15 \%$ of duodenoscopes intended to be ready for patient use. ${ }^{1}$ In a study from the USA that evaluated 4032 surveillance culture specimens from 106 duodenoscopes or curvilinear echoendoscopes, $5 \%$ showed some microbial contamination with $0.6 \%$ being pathogenic organisms. ${ }^{7}$

While it is obvious from collective evidence that the current combination of reprocessing and process control is insufficient, it is unclear whether a disposable duodenoscope represents the best solution. Our financial analysis reveals that in order to break-even, the cost must be set at $\$ 612$ per duodenoscope in our unit. However, this was estimated based on an assumed infection rate of $0.4 \%$; also, the treatment cost can vary based on the severity of illness, the type of hospital (public versus private) and between healthcare systems around the world. For instance, at our healthcare system, the hospital costs for a 2-day ICU stay was $\$ 125000$, which decreased to $\$ 50000$ if the care was provided in a non-critical care hospital setting. We encountered seven cases of postprocedure cholangitis in 2018, and duodenoscope cultures were negative for microbes in all seven instances. In a recent communication, the US Food and Drug Administration (FDA) announced interim findings from prospective evaluations that indicated higher-than-expected contamination rates after reprocessing, with up to $3 \%$ of properly collected samples testing positive for high concern organisms. ${ }^{8}$ However, it is unclear how frequently this microbial contamination is clinically relevant because a majority of post-ERCP infections are likely related to suboptimal ductal drainage and residual microbes; therefore, the incidence of infections directly attributed to duodenoscopes is unclear.

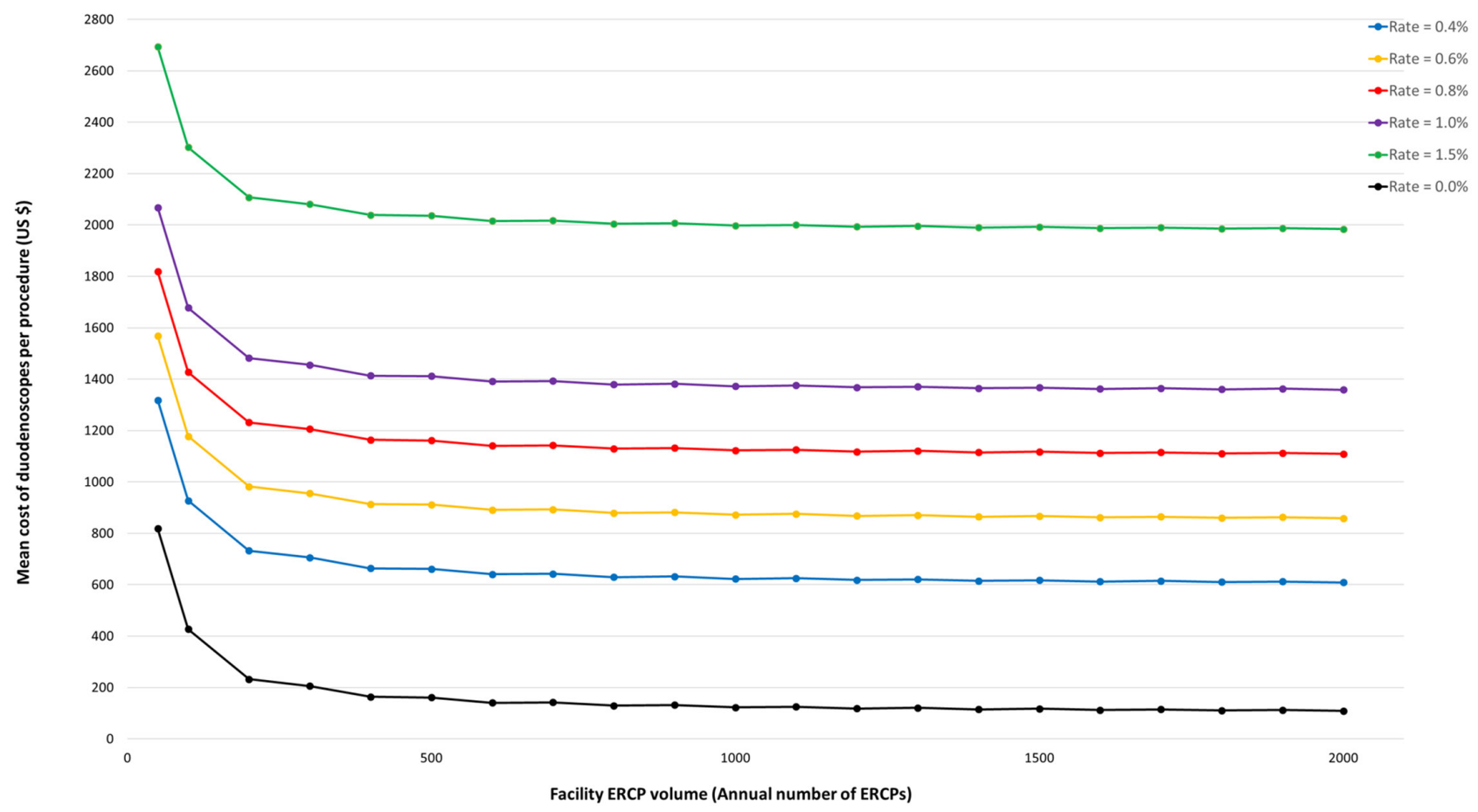

Figure 1 Line graph of the mean cost of duodenoscopes per procedure (US \$) according to infection rate and annual ERCP volume. ERCP, endoscopic retrograde cholangiopancreatography. 
What are the financial implications of a disposable duodenoscope being integrated into clinical practice? Currently, a reusable duodenoscope purchased for $\$ 35000$ is being used for 3 years to perform approximately 200 ERCPs per year at our unit, which can vary across centres. Should a disposable duodenoscope be substituted, it would incur a cost of $\$ 367200$ (\$612/ procedure), which is 10 times more than the present costs to treat the same number of patients. Borescope channel inspections of reprocessed endoscopes have revealed scratches, shredding and debris. ${ }^{9}$ The working channel is subject to wear and tear; the introduction of accessories creates complex surface area defects which allow bacteria to adhere and 'hide' from the cleaning process. It is unclear whether a relationship exists between the age of a duodenoscope and bacteria recovery rates. It will be interesting to culture and inspect for biofilm after each use of a reusable duodenoscopes to correlate the number of uses and the detection of bacterial contamination and biofilm. Depending on the results, it is possible that the most cost-effective strategy would be to use reusable duodenoscopes but limit the number of uses (for instance, every 60-80 procedures) and then completely refurbish the scope. Although this approach might appear impractical, it may well be less costly than a full conversion to disposable duodenoscopes. Furthermore, in addition to frequently scheduled servicing, it is possible that design changes and new methods of reprocessing could lower the risk of infection which would put further pressure on the cost point for single use duodenoscopes. Finally, if disposable duodenoscopes are to become popular, it is unclear if the costs will be covered by insurance carriers or if they will be passed on to patients. What seems clear is that a move to disposable duodenoscopes would add significant cost to the healthcare system.

What are the likely clinical implications of this new development? (1) Small volume institutions that do not want to invest in capital equipment but have the requisite technical expertise are more likely to use a disposable duodenoscope. Additionally, procedures that may have to be performed on an emergent basis outside of the endoscopy unit, such as the operating or emergency room, are likely to benefit given the ease of mobility and the lack of need to reprocess the endoscope after an offsite procedure. (2) Given the cost implications, conversion from reusable to disposable duodenoscopes may result in the concentration of ERCP services to large volume centres. (3) Although not quantifiable, the risk of transmitting colonised virulent microbes such as CRE can be eliminated, thereby reducing potentially exorbitant treatment costs and exposure to malpractice claims. (4) A recent study has shown that biliary stent placement, a diagnosis of cholangiocarcinoma and active inpatient status are associated with an increased risk of CRE transmission. ${ }^{2}$ One can, therefore, speculate that the use of a disposable duodenoscope may be relegated for performing ERCP only in such high-risk cases under specific circumstances.

While the development of a disposable duodenoscope represents a possible solution to transmission of infection during ERCP, the financial implications compel more data on their functionality and results from design changes, and new reprocessing methods for reusable duodenoscopes must be reviewed before their wider adoption into clinical practice.

Correction notice This article has been corrected since it published Online First. The last sentence of the Message section has been corrected.

Contributors JYB: interpretation of data, drafting of manuscript and critical revision of manuscript. SV: study concept and design, drafting of manuscript and critical revision of manuscript. BS: statistical analysis, drafting of manuscript and critical revision of manuscript. RH: critical revision of manuscript.

Funding The authors have not declared a specific grant for this research from any funding agency in the public, commercial or not-for-profit sectors.

Competing interests SV: consultant for Boston Scientific Corporation, Olympus America Inc, Covidien, Creo Medical. RH: consultant for Boston Scientific Corporation, Olympus America Inc, Covidien, Creo Medical, Nine Points Medical, Cook Medical.

Provenance and peer review Not commissioned; internally peer reviewed.

Open access This is an open access article distributed in accordance with the Creative Commons Attribution Non Commercial (CC BY-NC 4.0) license, which permits others to distribute, remix, adapt, build upon this work non-commercially, and license their derivative works on different terms, provided the original work is properly cited, appropriate credit is given, any changes made indicated, and the use is non-commercial. See: http://creativecommons.org/licenses/by-nc/4.0/.

\section{REFERENCES}

1 Rauwers AW, Voor In 't Holt AF, Buijs JG, et al. High prevalence rate of digestive tract bacteria in duodenoscopes: a nationwide study. Gut 2018;67:1637-45.

2 Kim S, Russell D, Mohamadnejad M, et al. Risk factors associated with the transmission of carbapenem-resistant Enterobacteriaceae via contaminated duodenoscopes. Gastrointest Endosc 2016:83:1121-9.

3 Alrabaa SF, Nguyen P, Sanderson R, et al. Early identification and control of carbapenemase-producing Klebsiella pneumoniae, originating from contaminated endoscopic equipment. Am J Infect Control 2013;41:562-4.

4 Epstein L, Hunter JC, Arwady MA, et al. New Delhi metallo- $\beta$-lactamase-producing carbapenem-resistant escherichia coli associated with exposure to duodenoscopes. JAMA 2014;312:1447-55.

5 Endoscopy presentation for the investment community at DDW. http://investors. bostonscientific.com/ /media/Files/B/Boston-Scientific-IR/documents/events/ endoscopy-investor-webcast-june-2018.pdf

6 Verfaillie CJ, Bruno MJ, Voor in 't Holt AF, et al. Withdrawal of a novel-design duodenoscope ends outbreak of a VIM-2-producing Pseudomonas aeruginosa. Endoscopy 2015;47:493-502.

7 Brandabur JJ, Leggett JE, Wang L, et al. Surveillance of guideline practices for duodenoscope and linear echoendoscope reprocessing in a large healthcare system. Gastrointest Endosc 2016:84:392-9.

8 The FDA provides interim results of duodenoscope reprocessing studies conducted in real-world settings: FDA safety communication. https://www.fda.gov/MedicalDevices/ Safety/AlertsandNotices/ucm628020.htm

9 Thaker AM, Kim S, Sedarat A, et al. Inspection of endoscope instrument channels after reprocessing using a prototype borescope. Gastrointest Endosc 2018;88:612-9. 